\title{
Patrones de distribución y abundancia del jurel Trachurus murphyi en el Perú
}

\author{
Abundance and distribution patterns of Jack mackerel Trachurus murphyi in Peru
}

\author{
Teobaldo Dioses
}

Instituto del Mar del Perú, esquina Gamarra y General Valle S/N Chucuito, Callao, Perú.

Email Teobaldo Dioses: tdioses@imarpe.gob.pe
Citación:

Dioses T. 2013. Patrones de distribución y abundancia del jurel Trachurus murphyi en el Perú. En: Csirke J., R. Guevara-Carrasco \& M. Espino (Eds.). Ecología, pesquería y conservación del jurel (Trachurus murphyi) en el Perú. Rev. peru. biol. número especial 20(1): 067- 074 (Septiembre 2013)

\section{Resumen}

Se analiza información de cruceros bio-oceanográficos del IMARPE con estaciones hasta 100 mn de la costa y entre 0 y 180 m de profundidad entre 1961 y 2011; información de observadores científicos a bordo de buques arrastrero-factoría de media agua nacionales, soviéticos y cubanos que operaron entre 1983 y 1998; y series de tiempo de anomalías de temperatura superficial del mar, profundidad de la isoterma de $15{ }^{\circ} \mathrm{C}$ y salinidad de 1950 a la fecha. Se identifican y describen tres patrones de distribución vertical y profundidad de las capturas de jurel Trachurus murphyi en relación a cambios en condiciones ambientales: (1) cuando ocurren fenómenos EI Niño (EN) fuertes los cardúmenes se distribuyen entre 100 y $200 \mathrm{~m}$ de profundidad, las capturas son altas y varían poco entre día y noche; (2) cuando hay fuertes afloramientos los cardúmenes se distribuyen entre 0 y 75 m de profundidad, la pesca es más oceánica y superficial y (3) cuando se refuerza la Extensión Sur de la Corriente de Cromwell (ESCC), los cardúmenes están entre 0 y $300 \mathrm{~m}$ de profundidad, la pesca es más costera, a mayor profundidad de día y más superficial de noche. T. murphyi es menos abundante en períodos fríos, cuando dominan Aguas Costeras Frías (ACF) y se alejan de la costa las Aguas Subtropicales Superficiales (ASS), y más abundante en periodos cálidos, asociados a eventos EN y dominancia de la ESCC. Se observa segregación latitudinal por tamaños en relación a la Oscilación Decadal del Pacífico.

Palabras claves: Jurel, distribución espacial, comportamiento, El Niño

\section{Abstract}

This paper analyzes information from IMARPE bio-oceanographic surveys conducted between 1961 and 2011 covering an area from 0 to $100 \mathrm{~nm}$ from the coast with observations at depths from 0 to $180 \mathrm{~m}$; information from scientific observers on board Peruvian, Soviet and Cuban factory trawlers operating between 1983 - 1998; as well as time series of sea surface temperature anomalies, depth of the $15{ }^{\circ} \mathrm{C}$ isotherm and sea surface salinity from 1950 to date. Three patterns in the vertical distribution and depth of catches of Jack mackerel Trachurus murphyi are identified and described in relation to changing environmental conditions: (1) During strong El Niño (EN) events shoals are at depths between $100-200$ m, catches are higher and don't change much between day and night; (2) During strong upwellings shoals are shallower, at depths between $0-75 \mathrm{~m}$ and catches are more oceanic (offshore) and closer to the surface; and, (3) When there is a strengthen Southern Extension of the Cromwell Current (SECC) shoals are at depths between 0 and $300 \mathrm{~m}$, fishing takes place closer to the coast being deeper during the day and shallower during the night. During cold periods there is lower abundance of $T$. murphyi due to the prevalence of Cold Coastal Waters (CCW) and the offshore shift of the Subtropical Surface Waters (SSW), while abundance is higher during warmer periods associated with EN events and the strengthening of the SECC. A certain latitudinal segregation by sizes in relation to the Pacific Decadal Oscillation is also observed.

Keywords: Jack mackerel, spatial distribution, behavior, El Niño 


\section{Introducción}

El ambiente marino entre los $04^{\circ} \mathrm{S}$ y el extremo sur del dominio marítimo peruano está dominado por el Sistema de la Corriente del Perú (SCP), que sustenta una alta producción pesquera y que recurrentemente se ve afectada por fuertes variaciones del clima y de las condiciones oceanográficas en ciclos o estadios superpuestos de amplitud diversa. Estas variaciones pueden ser de corta duración o estacionales, de mediano plazo como los eventos ENSO (El Niño-Oscilación del Sur) con sus fases cálidas (El Niño) y frías (La Niña), los de largo plazo con periodos decadales cálidos y fríos de 25 años a más (Chávez, et al. 2003, Chávez et al. 2008); y por ultimo otros seculares (mayores a 50 años) (Espino 2003, Espino \& Yamashiro 2012).

La distribución y concentración de Trachurus murphyi Nichols 1920 se relacionan con la variación e interacción de las masas de agua que existen frente a la costa peruana, según se trate de ańos normales, o anormales con calentamiento (El Niño) o enfriamiento (La Niña). El hábitat preferido de T. murphyi es el frente oceánico formado por las Aguas Costeras Frías (ACF) y las Aguas Subtropicales Superficiales (ASS). Los frentes y remolinos que se forman entre estas masas de agua tendrían un efecto sobre algunas características biológicas y de comportamiento de T. murphyi (áreas y épocas de reproducción, sobrevivencia de larvas, cambios en distribución y disponibilidad, entre otros). La abundancia y la disponibilidad de este recurso se explican por las variaciones de este frente.

En este trabajo se describe y analiza las concentraciones de los cardúmenes del jurel T. murphyi con los cambios ambientales de corto y mediano plazo que se han presentado durante el desarrollo de esta pesquería.

\section{Material y métodos}

Los datos analizados fueron obtenidos de los cruceros realizados por el IMARPE frente a la costa del Perú, en una malla de estaciones bio-oceanográficas distribuidas hasta las $100 \mathrm{mn}$ de la costa y secciones oceanográficas verticales hasta los 180 $\mathrm{m}$ de profundidad estándar, en el periodo comprendido entre 1961 - 2011. También se analizó la información obtenida por los Técnicos Científicos de Investigación (TCI) a bordo de buques arrastrero-factoría (BAF) de media agua nacionales (1996 - 1998), soviéticos (1983 - 1987) y cubanos (1988 - 1991) que han operado en la pesca de T. murphyi en aguas peruanas. Además se analizaron series de tiempo de 1950 a la fecha de las anomalías de la temperatura superficial del mar (ATSM), de la profundización de la isoterma de $15^{\circ} \mathrm{C}$ y de la salinidad.

En función de los datos tomados por los TCI, tales como fecha, área de pesca, según los cuadrados Marsden ( $1^{\circ}$ Lat. x $1^{\circ}$ Long.) e información de los arrastres (hora, posición, rumbo, profundidad de la red, captura estimada, temperatura del agua y del aire, etc.), Icochea et al. (1989) analizaron el periodo febrero 1983 a marzo 1987 y Chirinos (1992) analizó el periodo mayo 1988 a abril 1991. Estos investigadores estudiaron las operaciones de pesca en que se capturaron especies pelágicas, con especial énfasis en el jurel. $\mathrm{El}$ área de estudio estuvo comprendida entre los $05^{\circ} \mathrm{S}$ y los $09^{\circ} \mathrm{S}$. El límite norte de operación de la flota arrastrera determinada por el Gobierno Peruano fue los $06^{\circ} \mathrm{S}$, para el periodo febrero 1983 - marzo 1985, y a partir de abril de 1985 fue los $05^{\circ} \mathrm{S}$. Dioses et al. (1989) explican la distribución batimétrica de los cardúmenes de T. murphyi en desove entre octubre y diciembre de 1986.
El periodo 1996 - 2007 fue analizado por Espinoza, Niquen y Flores (2008) y el periodo 2008 - 2012 por Espinoza (com. pers.) en base a la información del Programa Bitácoras de Pesca. Este Programa registra las operaciones a bordo de la flota de cerco autorizada para la pesca de T. murphyi a nivel industrial, que es una flota equipada con sistema de enfriamiento por circulación de agua de mar (RSW).

Se establecieron patrones de comportamiento migratorio vertical y horizontal del recurso mediante la revisión y análisis de los ecogramas más representativos, de la distribución horizontal y batimétrica de los cardúmenes en relación al ambiente.

Para el análisis de la relación recurso-ambiente se presenta la distribución vertical de los cardúmenes de T. murphyi asociada con el ambiente (temperatura, salinidad, oxígeno), en lances de pesca seleccionados.

\section{Resultados y discusión}

En el mar peruano, el hábitat preferido por el T. murphyi es el frente oceánico formado por las Aguas Costeras Frías (ACF) con las Aguas Subtropicales Superficiales (ASS). En tales condiciones, los cardúmenes se ubican en la columna de agua con rangos de temperatura entre 14 y $24{ }^{\circ} \mathrm{C}$, Ganoza (1998). En relación a la profundidad, conforme más cerca de la costa, las isotermas adoptan una configuración de divergencia vertical, unas por encima de la termoclina y que se dirigen hacia la superficie (afloramiento costero) y otro grupo de isotermas por debajo de la termoclina que se profundizan y que están asociadas a la ESCC. La profundidad de la isoterma de $15^{\circ} \mathrm{C}$ constituye el valor inmediato por debajo de la termoclina y es la que marca la profundidad donde se produce la divergencia de las isotermas (Flores et al. 2009).

De la información anterior y de los trabajos de Icochea et al. (1989), Chirinos (1992), Ganoza (1998) y Dioses et al. (1989) se describen los siguientes patrones de comportamiento del $T$. murphyi frente a su ambiente:

El primer patrón se distingue durante la ocurrencia del fenómeno El Niño, cuando las especies pelágicas se concentran debajo de una marcada y profunda termoclina. Las capturas se realizan a profundidades mayores de $100 \mathrm{~m}$, en temperaturas de 15 a $20^{\circ} \mathrm{C}$, salinidades entre 35.0 - 35.1 ups y niveles de oxígeno mayores a $1 \mathrm{~mL} / \mathrm{L}$. Bajo estas condiciones las capturas tienden a ser altas y no varían significativamente durante el día debido a la reducción del rango de distribución de los cardúmenes. La presencia de elevadas temperaturas superficiales, por encima de los $29^{\circ} \mathrm{C}$, cinco grados más sobre lo normal y de una marcada termoclina que llega hasta los $200 \mathrm{~m}$, determinan que los cardúmenes de T. murphyi se presenten a profundidades mayores a $100 \mathrm{~m}$, entre 15 y $20^{\circ} \mathrm{C}$. Las capas superficiales del mar son ocupadas por las Aguas Ecuatoriales Superficiales (AES) y las Aguas Tropicales Superficiales (ATS) de altas temperaturas y bajas salinidades. En estas condiciones el recurso es más accesible y vulnerable a la flota de arrastre que a la cerquera (Fig. 1a y Fig. 2a y b).

El segundo patrón se suele observar posterior a los eventos El Nińo, con dominio de las ACF. Las isotermas mayores a 15 ${ }^{\circ} \mathrm{C}$ se encuentran más superficiales conforme más cerca de la costa, con salinidades mayores a 34.95 ups, y concentraciones de oxígeno entre 1 y $3 \mathrm{~mL} / \mathrm{L}$. (Fig. 1 b y Fig. $3 \mathrm{a}$, b y c). La captura es más superficial y oceánica, fluctuando con la extensión del 


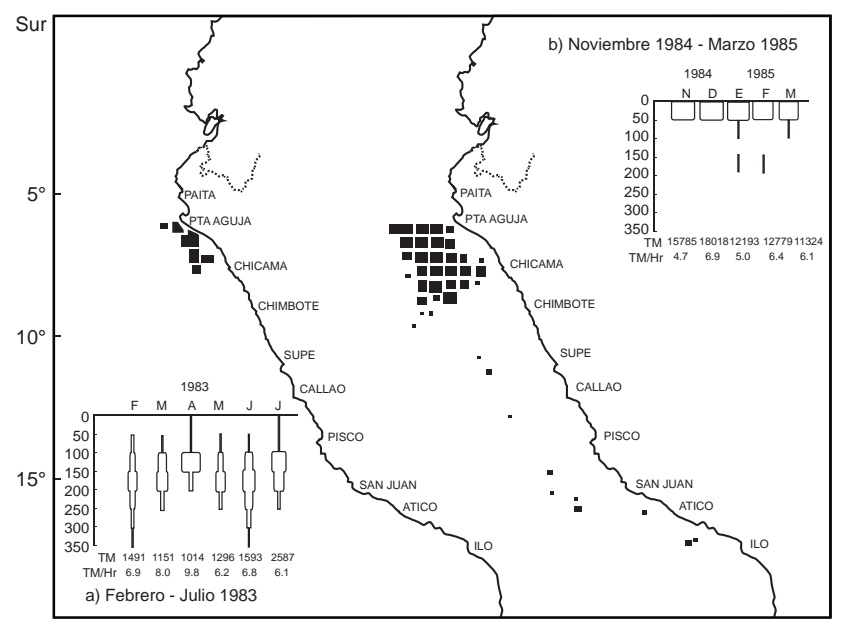

Figura. 1 Principales zonas de pesca y distribución vertical de las capturas de jurel T. murphyi: a) Periodo El Niño febrero - julio 1983, b) Periodo noviembre 1984 - marzo 1985. (Modificada de Icochea et al. 1989).

Figure. 1 Major fishing areas and vertical distribution of Jack mackerel T. murphyi: a) El Niño Period February to July, 1983, b) Period November 1984 - March 1985. (Modified after Icochea et al. 1989).

frente oceánico. En este segundo patrón, el valor de $1.0 \mathrm{~mL} / \mathrm{L}$ de oxígeno se ubica aproximadamente a los $50 \mathrm{~m}$ de profundidad, lo que explica la presencia superficial de los cardúmenes de $T$. murphyi y la ausencia de este recurso a mayores profundidades.

El tercer patrón se observa cuando se refuerza la ESCC, detectada por la profundización a niveles cercanos a los $300 \mathrm{~m}$ de las isotermas de $14^{\circ} \mathrm{C}$ y $15^{\circ} \mathrm{C}$ cerca de la costa. Trachurus murphyi es capturado a profundidades mayores a $100 \mathrm{~m}$ en el día y cerca de la superficie durante la noche. Las capturas alcanzan valores más altos en el día, debido a que en la noche se encuentra más disperso en la superficie debido a la dispersión de su alimento (Fig. 4). Además se observan migraciones verticales diurnas hasta $300 \mathrm{~m}$ de profundidad favorecidas por la ausencia de una marcada termoclina y la presencia de aguas con alto contenido de oxígeno disuelto a mayores profundidades. El valor de oxígeno de 1,0 mL/L alcanza profundidades cercanas a los $300 \mathrm{~m}$ y ocurre lo mismo con la isoterma de $14^{\circ} \mathrm{C}$. En este caso, los cardúmenes prefieren rangos de temperatura entre 15 y $20^{\circ} \mathrm{C}$, contenido de oxígeno entre 1.0 y $5.0 \mathrm{~mL} / \mathrm{L}$ y de salinidad de 35.0 ups (Fig. 5).

Mediante la comparación de la distribución batimétrica de los cardúmenes en desove (con ovocitos hidratados), con los parámetros oceanográficos (temperatura, salinidad y oxígeno) de

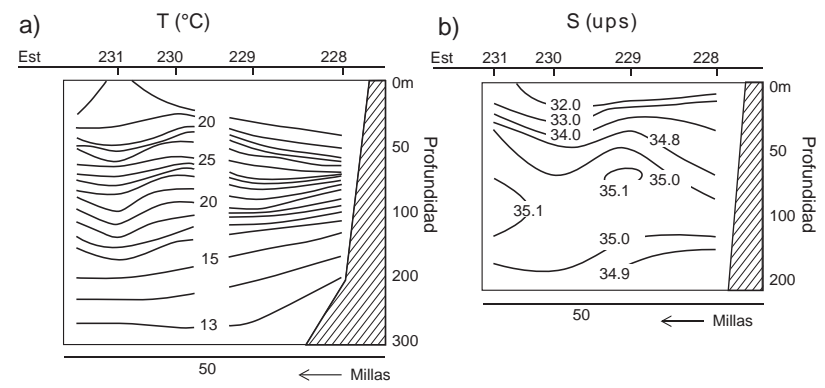

Figura 2.- Distribución vertical de a) Temperatura y b) Salinidad frente a Punta Aguja en febrero - marzo 1983. (de Icochea et al. 1989)

Figure 2. - Vertical distribution of a) temperature and b) salinity, off Punta Aguja in February-March 1983. (from Icochea et al. 1989)
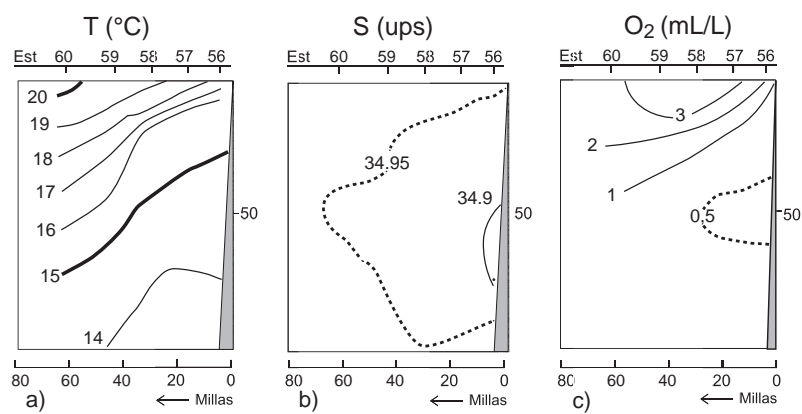

Figura 3.- Distribución vertical de a) Temperatura, b) Salinidad y c) Oxígeno frente a Punta Aguja en diciembre 1984. (de Icochea et al. 1989)

Figure 3. - Vertical distribution of a) temperature, b) salinity and c) off Punta Aguja Oxygen in December 1984. (from Icochea et al. 1989)

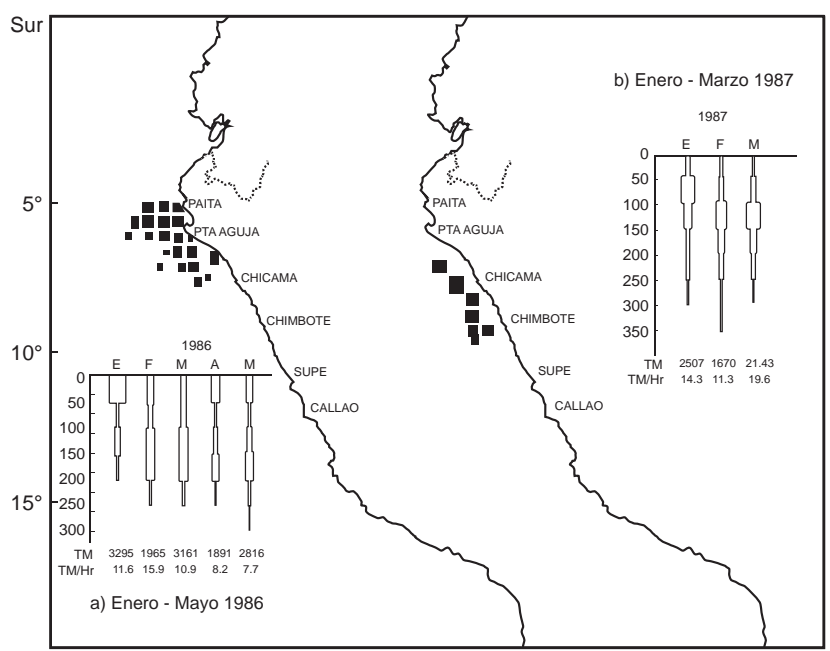

Figura 4. Principales zonas de pesca y distribución vertical de las capturas de jurel T. murphyi a) Periodo enero - mayo 1986, b) Periodo enero - marzo 1987. (Modificada de Icochea et al. 1987)

Figure 4. Main fishing areas and vertical distribution of Jack mackerel T. murphyi: a) Period January to May 1986, b) Period January to March 1987. (Modified from Icochea et al. 1987)

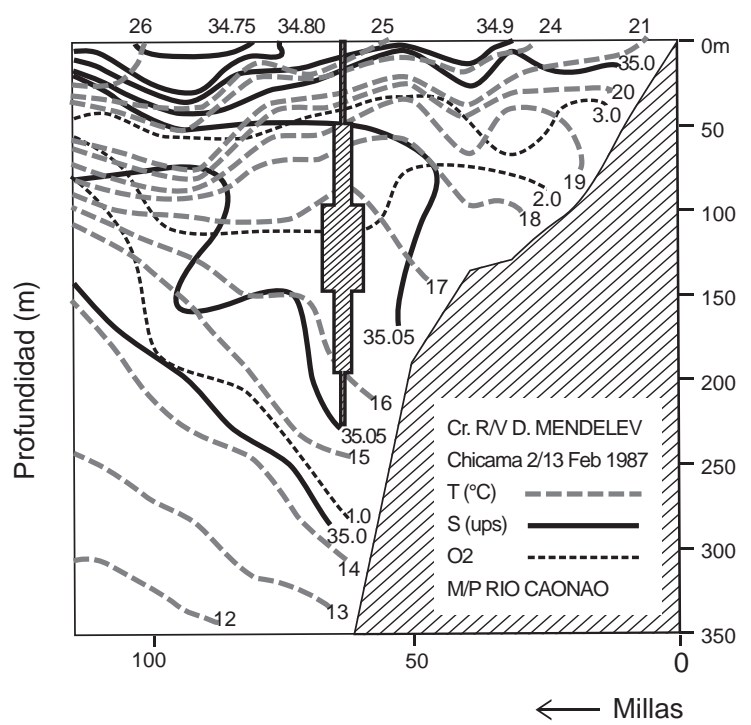

Figura 5.- Distribución vertical de los cardúmenes de jurel T. murphyi, en relación con los parámetros oceanográficos de temperatura, salinidad y oxígeno frente a Chicama (2/13 Febrero 1987).

Figure 5.- Vertical distribution of Jack mackerel T. murphyi schools in relation to oceanographic parameters of temperature, salinity and oxygen off Chicama (February 2/13, 1987). 


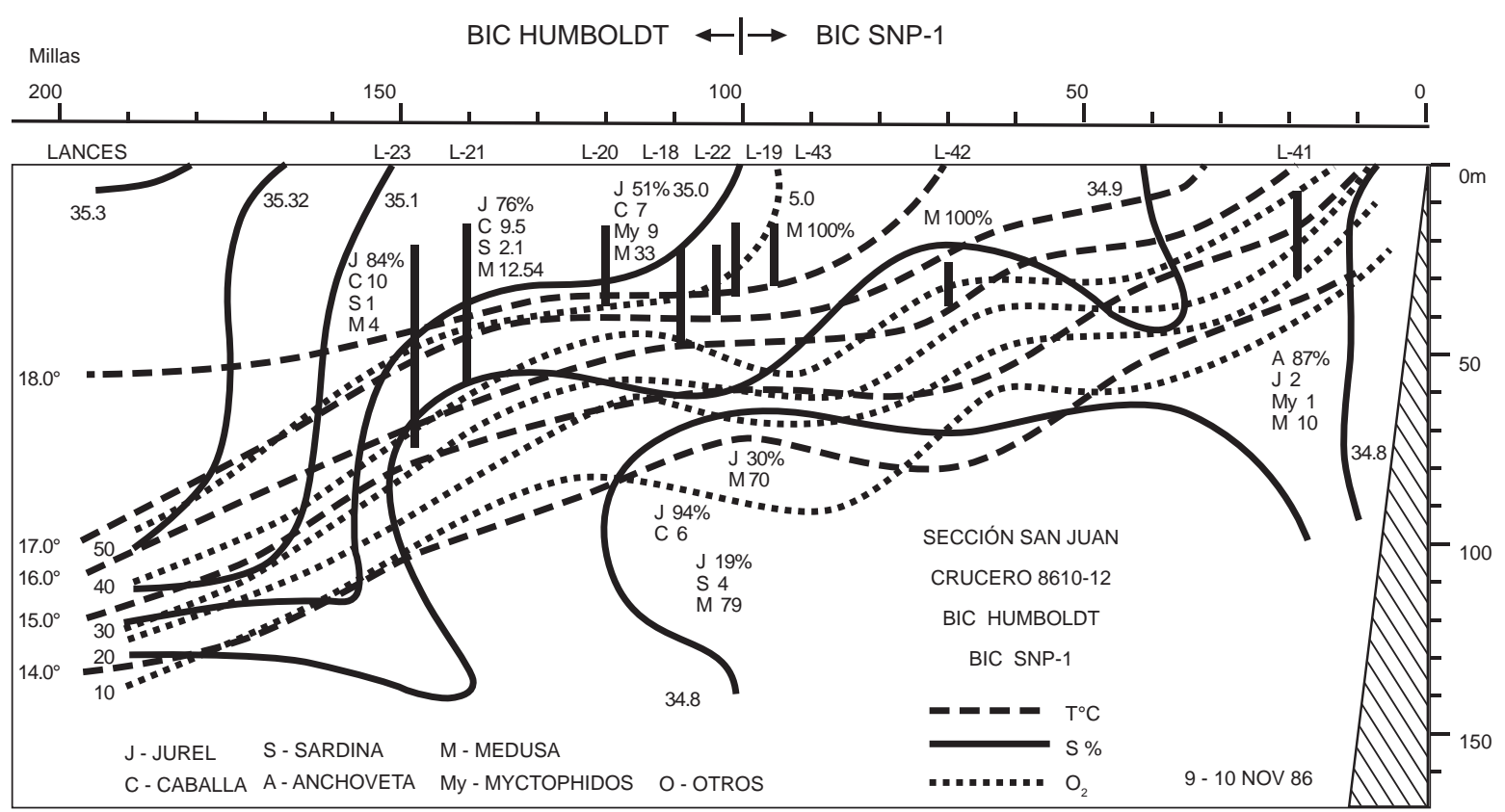

Figura 6.- Distribución vertical de los cardúmenes en desove de jurel T. murphyi, en relación con los parámetros oceanográficos de temperatura, salinidad y oxígeno frente a San Juan (9/10 noviembre 1986) (de Dioses et al. 1989).

Figure 6.- Vertical distribution of Jack mackerel T. murphyi spawning schools in relation to oceanographic parameters of temperature, salinity and oxygen off San Juan (November, 9/10, 1986) (from Dioses et al. 1989).

la sección San Juan (Cr. 8610-12), Dioses et al. (1989) determinaron que los cardúmenes de T. murphyi en desove se localizan entre las 100 y 150 millas de la costa, en el frente delimitado por las ACF, producto del intenso afloramiento de Punta San Juan (salinidades menores de 35.0 ups) y las ASS (salinidades mayores a 35.1 ups), con temperaturas y concentraciones de oxígeno mayores a $18{ }^{\circ} \mathrm{C}$ y $5,0 \mathrm{~mL} / \mathrm{L}$ respectivamente. (Fig. 6)

La característica del desove de T. murphyi, de efectuarse en la capa superficial, sobre los $50 \mathrm{~m}$ y de requerir altos valores de oxigeno determinan que los cardúmenes en desove de T. murphyi sean pequeños y estén dispersos entre el dominio marítimo sur de Perú y los $14^{\circ} \mathrm{S}$. Por ser esta especie desovadora de primavera no tiene mayor interacción con los desoves de anchoveta Engraulis ringens, sardina Sardinops sagax y caballa Scomber japonicus, por ocupar diferentes áreas de desove.

El desove en esta área dentro de la zona eufótica y por encima de los $50 \mathrm{~m}$ permite que las larvas de T. murphyi sean desplazadas hacia la zona del frente de las ACF con las ASS por efecto del intenso transporte de Ekman (fenómeno de deriva larvaria). Este frente se despliega por fuera de las $90 \mathrm{mn}$ de la costa en julio, agosto y setiembre principalmente (Santander \& Flores 1983). Esto también explica la disponibilidad estacional de este recurso cuando el frente oceánico se acerca a la costa, que lo hace más accesible a la pesquería.

Por otro lado, se ha observado que entre la isoterma de $15^{\circ} \mathrm{C}$ y el contenido de $1.0 \mathrm{~mL} / \mathrm{L}$ de oxígeno existe una importante relación que sirve como indicador de la presencia de esta especie para la flota pesquera. Sobre la base de este valor de contenido de oxígeno, cercano al mínimo $(0.5 \mathrm{~mL} / \mathrm{L})$, se han esquematizado los tres patrones de comportamiento de la distribución vertical de los cardúmenes de T. murphyi (Fig. 7). Para el patrón 1, se observa que el tenor de $1.0 \mathrm{~mL} / \mathrm{L}$ de oxígeno alcanza los $200 \mathrm{~m}$ de profundidad y los cardúmenes se ubican entre esta profundidad y los $100 \mathrm{~m}$, como ocurre durante los eventos El Niño fuertes como en 1983. En el patrón 2, de fuertes afloramientos, el valor de $1.0 \mathrm{~mL} / \mathrm{L}$ se halla sobre los $100 \mathrm{~m}$ de profundidad y los cardúmenes se presentan sobre los $75 \mathrm{~m}$. En el patrón 3, característica de una marcada presencia de la ESCC (en ausencia de un evento El Niño o en presencia de eventos suaves o moderados), el valor de $1.0 \mathrm{~mL} / \mathrm{L}$ alcanza profundidades cercanas a los $300 \mathrm{~m}$ y permite una amplia migración de los cardúmenes entre esta profundidad y la superficie.

La variabilidad ambiental de largo plazo afecta la distribución y abundancia del T. murphyi a lo largo del mar peruano, tal como se puede observar en la distribución latitudinal de norte (Paita) a sur (Ilo) de las anomalías de la TSM entre 1950 y 2010 (Fig. 8). Además, el periodo cálido (1972 - 1998) es el más apto para la presencia de altas biomasas de T. murphyi, en

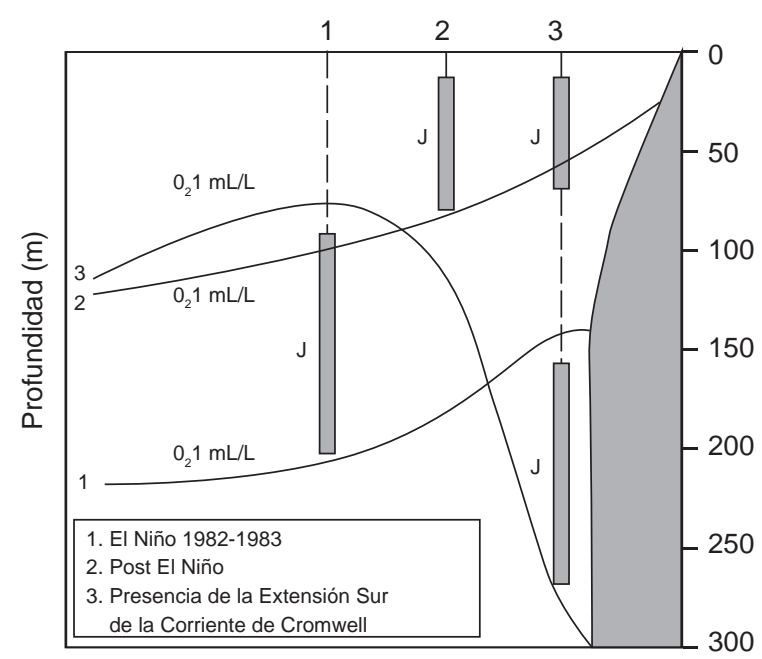

Figura 7. Patrones de distribución vertical de jurel T. murphyi en relación a la isooxígena de $1 \mathrm{~mL} / \mathrm{L}$ (Modificada de Chirinos.1992)

Figure 7. Vertical distribution patterns Jack mackerel T. murphyi in relation to oxygen isoline of $1.0 \mathrm{~mL} / \mathrm{L}$ (Modified from Chirinos. 1992) 


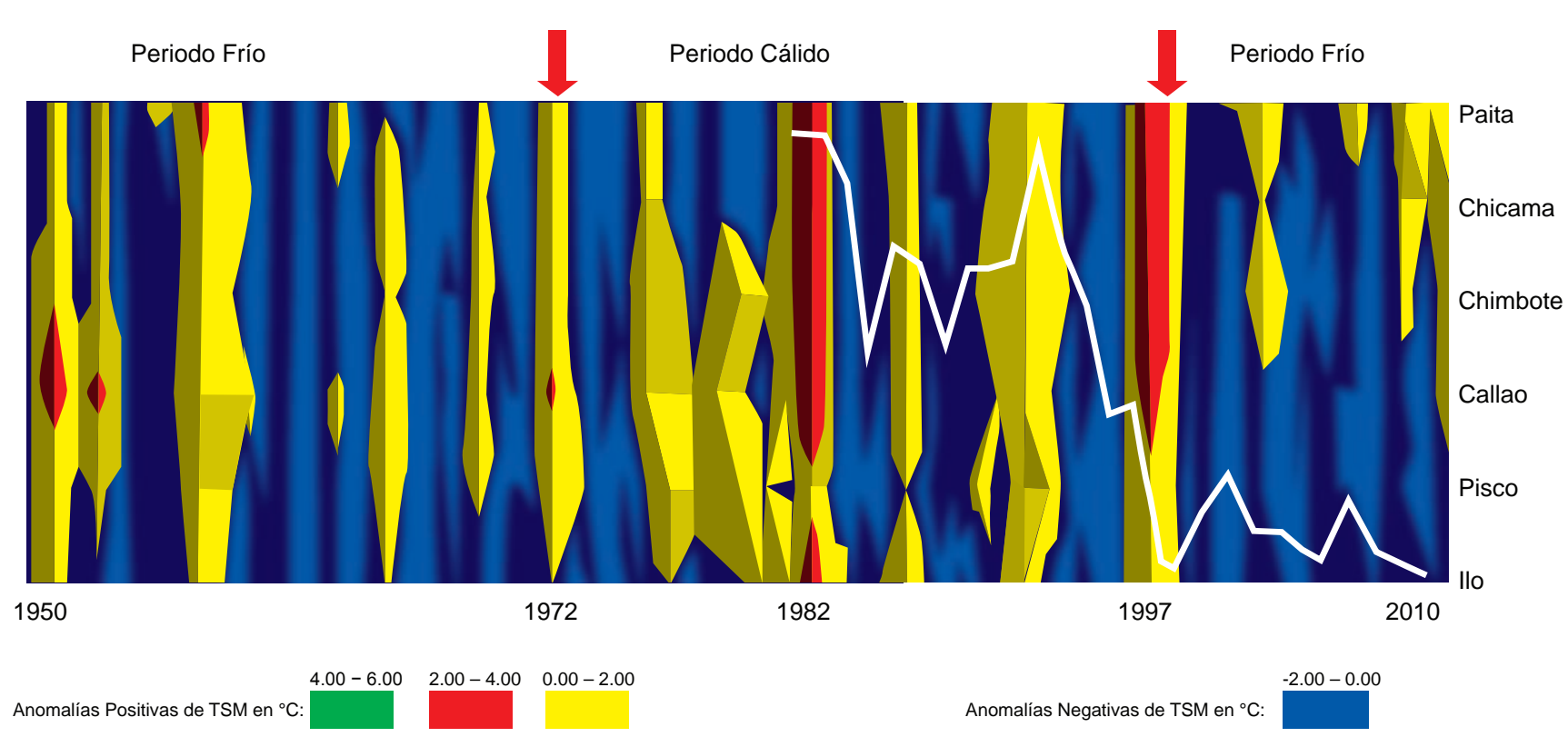

Figura 8. Anomalías de la temperatura superficial del mar de Paita a llo (1950 - 2010) y biomasas acústicas de jurel T. murphyi (1983 - 2010). Figure 8. Sea surface temperature anomalies from Paita to llo (1950 - 2010), and Jack mackerel T. murphyi acoustic biomass (1983 - 2010).

el cual destacan las mayores abundancias en años de El Niño, con notables disminuciones en años fríos.

Durante el periodo cálido (1972 - 1998) se presentaron los tres patrones de distribución, en tanto que en el actual periodo frío, se ha observado principalmente el patrón 2.

En cuanto a la variabilidad de la profundización de la isoterma de $15^{\circ} \mathrm{C}$, como indicador de flujos dirigidos hacia el sur, Flores et al. (2013) mencionan que las mayores profundizaciones se han producido durante los eventos El Niño 1982-83 y 1991-92, tanto al norte, centro y sur de la costa peruana. Esto nos indica una presencia fuerte de la ESCC, la cual favorece la disponibilidad y abundancia del jurel durante estas etapas. Después de estos eventos, los flujos dirigidos hacia el sur se repliegan hacia el norte. En este proceso se produce una disminución en la abundancia y disponibilidad de este recurso.

El acercamiento a la costa de las ASS (Flores et al. 2013) durante periodos cálidos de largo plazo (Espino \& Yamashiro 2012, Chávez et al. 2003) favorece la presencia, disponibilidad y abundancia del T. murphyi. Eso se aprecia en las altas biomasas de la década de los 80 y mediados de los 90. A partir del año 2001, entre los 08 y $14^{\circ} S$, se produce una mayor disponibilidad de jurel, probablemente debido al acercamiento de las ASS a esta área y a la presencia de las AES al norte de los $08^{\circ}$ S. (Fig. 9)
El análisis de la estacionalidad de las biomasas acústicas de $T$. murphyi por grados de latitud durante el periodo cálido 1972 - 1998 muestra un desplazamiento norte-sur desde el verano hasta la primavera (Fig. 10). Durante el verano, las mayores biomasas se ubican en el norte $\left(05-11^{\circ} \mathrm{S}\right)$ mientras que en otońo se extienden tanto al norte, centro y sur. En invierno se observa una tendencia a ser más abundante en el sur y en primavera se aprecia una mayor abundancia en la zona centro-sur, que coincide con la época principal del desove. Se destaca que al sur de los $14^{\circ} \mathrm{S}$ se encuentra una de las principales áreas de desove de T. murphyi en el mar peruano.

Después de El Niño 1997-98 a la fecha se viene experimentando a lo largo del mar peruano lo que se considera un periodo frío, durante el cual se observa un desplazamiento de las concentraciones de biomasa por latitud y estacionalmente hacia el sur de los $06^{\circ} \mathrm{S}$. Los mayores valores de biomasa latitudinales se presentan en la zona centro-sur, especialmente al sur de los $11^{\circ} \mathrm{S}$, con los valores más bajos en la estación de otońo (Fig. 11).

Dioses y Niquen (1988) observaron el aumento latitudinal, de sur a norte, más o menos gradual en la estructura por tamańos del T. murphyi en las capturas de muestreo realizadas durante un crucero científico en agosto-setiembre 1987 (Fig. 12B). Posteriormente Dioses (1995) observó algo semejante en las capturas comerciales realizadas en enero-mayo 1990 (Fig. 12A). En ambos

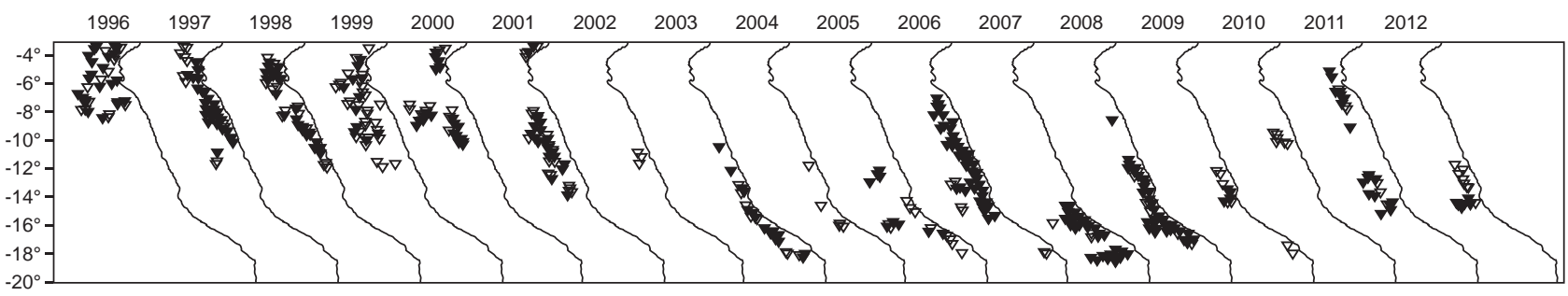

Figura 9. Distribución de las posiciones geográficas de las capturas de jurel T. murphyi registradas a bordo, durante las actividades de pesca de la flota de cerco entre 1996 - 2007. (Espinoza et al. 2008)

Figure 9. Geographic distribution of recorded catches of Jack mackerel T. murphyi, on board the purse seine fleet, between 1996 and 2007. (Espinoza et al. 2008) 


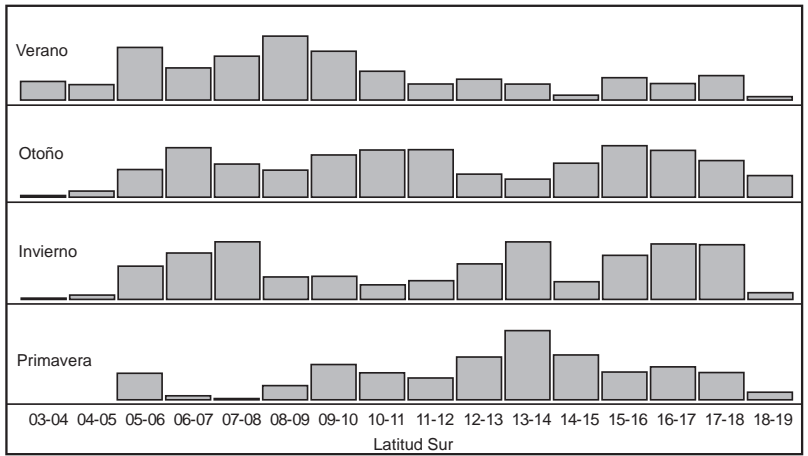

Figura. 10 Biomasa acústica de jurel T. murphyi por grados de latitud y por estaciones durante el periodo cálido 1972 - 1998.

Figure. 10 Acoustic biomass of Jack mackerel T. murphyi by latitude, during the warm period $1972-1998$.

casos se observa que en la zona sur las capturas estuvieron compuestas por especímenes menores a $35 \mathrm{~cm}(20-35 \mathrm{~cm})$ y por especímenes de mayor tamaño $(33-62 \mathrm{~cm})$ en la zona norte.

La información de la flota arrastrera pelágica extranjera analizada entre 1983 y 1991 nos muestra que esta flota extrajo T. murphyi desde los $05^{\circ} \mathrm{S}$ hasta los $15^{\circ} \mathrm{S}$ y entre 10 y $200 \mathrm{mn}$ de distancia de la costa, tal como se puede observar en la distribución de las áreas de pesca del T. murphyi mediante áreas isoparalitorales de 1988 a 1991 (Fig. 13).

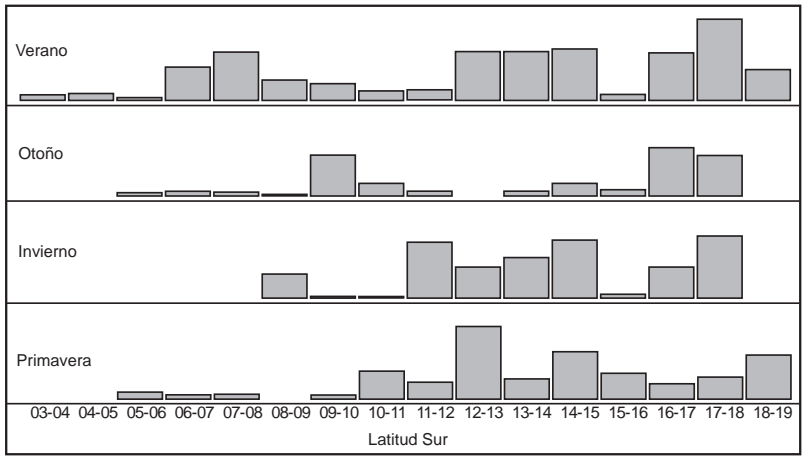

Figura. 11 Biomasa acústica de jurel T. murphyi por grados de latitud y estaciones durante el periodo frio $1999-2008$.

Figure. 11 Acoustic biomass of Jack mackerel T. murphyi by latitude, during the cold period $1999-2008$.

De abril a agosto 1989 las áreas de pesca se extendieron desde el grado 06 al sur hasta alcanzar los $12^{\circ} \mathrm{S}$ en el mes de mayo. La distancia promedio de la costa fue $70 \mathrm{mn}$. Durante junio y julio, la flota se concentró entre los 06 y $08^{\circ} \mathrm{S}$ y se extendió hacia el oeste $(200 \mathrm{mn})$. La profundidad de pesca alcanzó los $200 \mathrm{~m}$ en las áreas más cercanas a la costa y se hizo superficial en las zonas más alejadas. Este panorama fue debido a una nueva incursión de la Corriente de Cromwell cuya influencia alcanzó aproximadamente las $70 \mathrm{mn}$ de la costa y los $200 \mathrm{~m}$ de profundidad. En
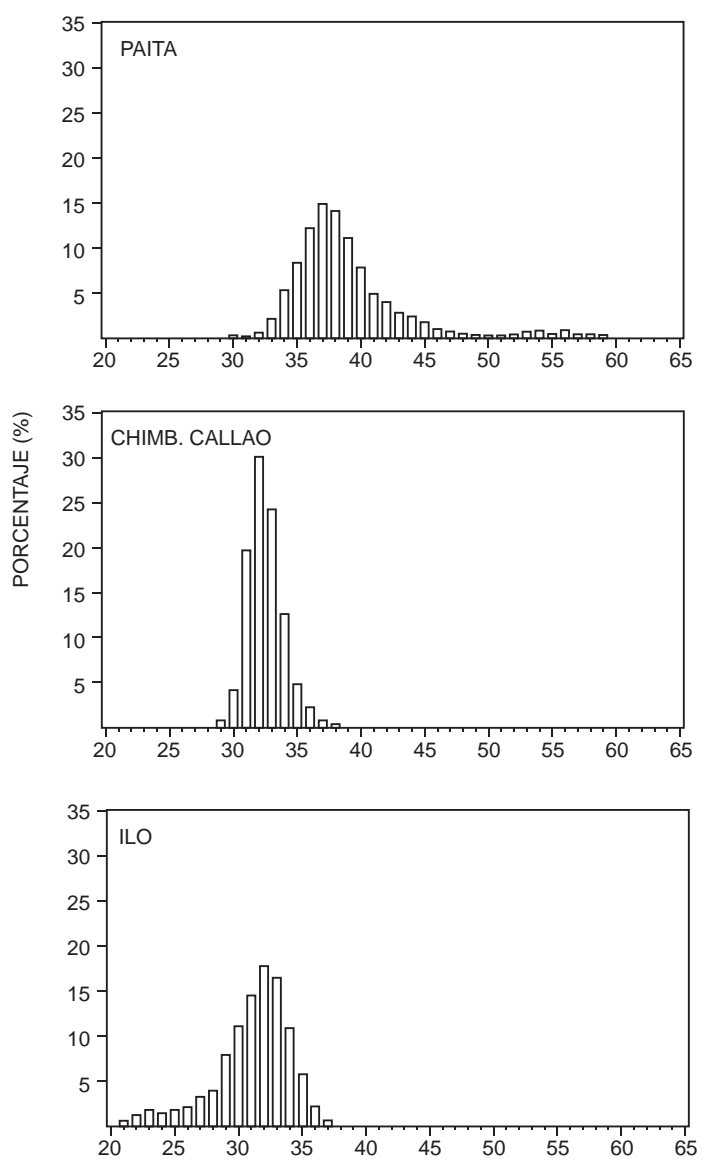

(A)

Longitud total $(\mathrm{cm})$

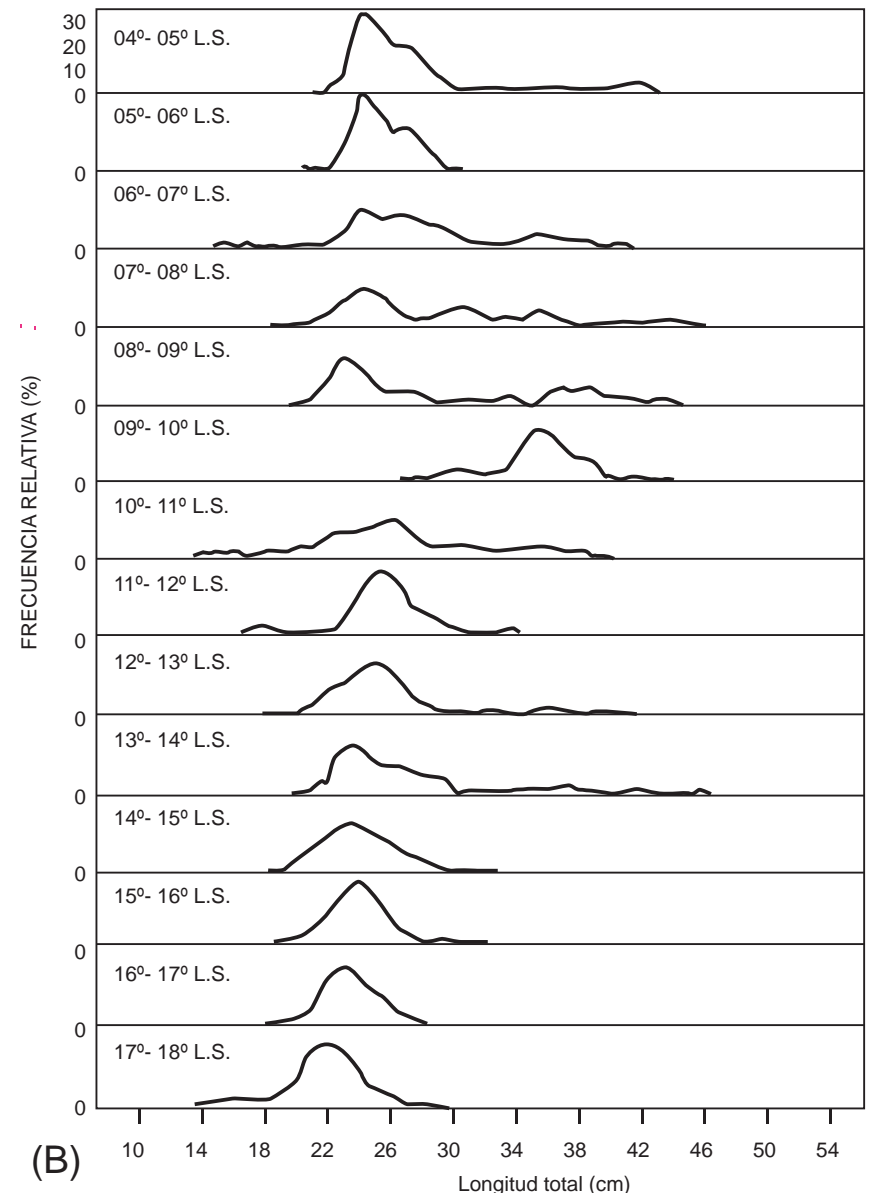

(B)

Figura 12. Patrones de la estructura por tallas de jurel T. murphyi (A): en la pesquería (enero a mayo 1990) (Dioses 1995), y (B) durante el crucero, BIC HUMBOLDT y SNP-1 8708-09 (redibujado de Dioses \& Ñiquen 1988).

Figure 12. Patterns of size structure of Jack mackerel T. murphyi (A): in the fishery (January-May 1990) (from Dioses 1995), and (B) during the scientific survey, BIC HUMBOLDT and SNP-1 8708-09 (redrawn after Dioses \& Ñiquen 1988). 


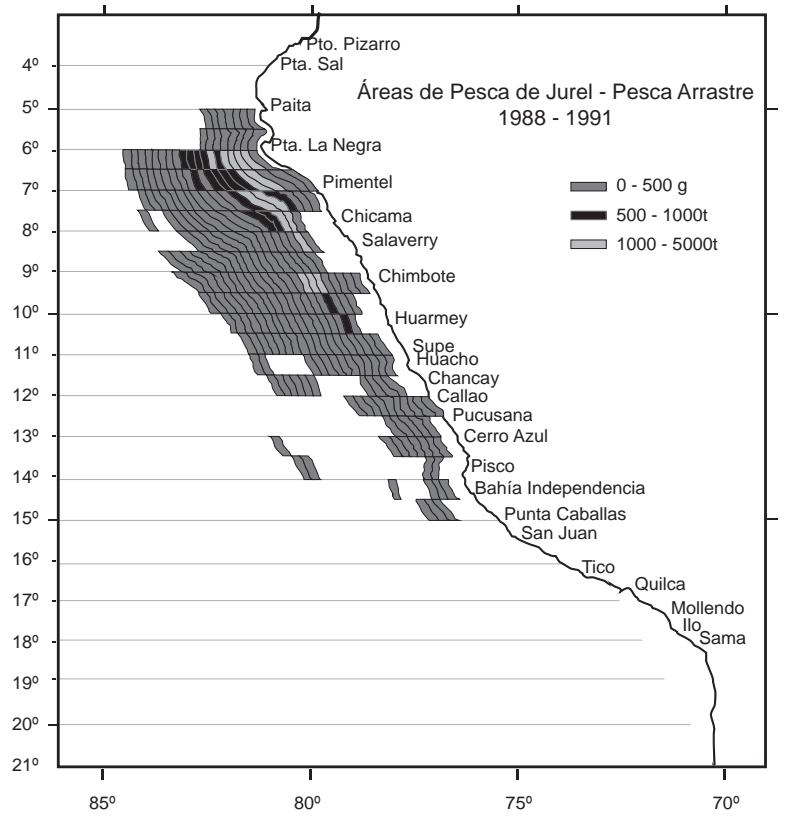

Figura 13. Áreas de pesca de jurel T. murphyi de la flota de arrastre pelágico (abril 1988 - mayo1991).

Figure 13. Jack mackerel T. murphyi fishing areas of the midwater trawl fleet (April 1988 - May1991).

setiembre-octubre de 1989 la pesca fue mayormente superficial, favorecida por un intenso afloramiento.

Entre julio 1990 y febrero de 1991 ocurrió la distribución más amplia de todo el período analizado, del grado $06^{\circ} \mathrm{S}$ al $15^{\circ} \mathrm{S}$. Trachurus murphyi estuvo bastante disperso entre las 30 y $200 \mathrm{mn}$ de distancia a la costa. La ESCC se presentó entre Punta Falsa y Paita debilitada por el proceso de enfriamiento del invierno.

$\mathrm{Al}$ sur de los $15^{\circ} \mathrm{S}$ esta flota no operó, porque esta área es la zona habitual de desove y de cría de esta especie, siendo por lo tanto los jureles de esta zona pequeños y no rentables para este tipo de barcos factoría.

Es importante destacar que estas observaciones (estructura por tamaños y zona de desove) fueron realizadas entre los años 1972 y 1997, periodo decadal cálido (PDO+) y que muestran cierta segregación espacial por tamaños, con los especímenes más pequeños en el sur, lo que sugiere una migración hacia el norte durante el ciclo de vida de esta especie.

Actualmente, después de El Niño 1997-98, estamos viviendo un período decadal frío (PDO-), período en el cual venimos observando fuertes afloramientos con valores de oxigeno de $1 \mathrm{~mL} / \mathrm{L}$ desde $80-100 \mathrm{~m}$ de profundidad a las $90 \mathrm{mn}$ de distancia a la costa hasta $20-30 \mathrm{~m}$ de profundidad cerca a la costa. Con frentes apropiados para la captura de T. murphyi entre las $40-60 \mathrm{mn}$. Esta es una de las razones por la cual el $T$. murphyi se ubica más superficial en la actualidad, estando más disponible y accesible a la flota cerquera. Esta flota con sistema de refrigeración RSW y dedicada a su captura viene operando entre los $05^{\circ} \mathrm{S}$ y los $17^{\circ} \mathrm{S}$ y desde las 10 a $110 \mathrm{mn}$ de la costa.

Entre junio y diciembre de 1998 debido a un fuerte afloramiento el recurso se ubicó entre 05 y $08^{\circ} \mathrm{S}$, concentrándose en el grado $06^{\circ} \mathrm{S}$. En junio - julio la flota operó dentro de las $70 \mathrm{mn}$, para luego desplazarse hacia áreas más alejadas alcanzando las $150 \mathrm{mn}$. La profundidad de pesca estuvo sobre los $100 \mathrm{~m}$ en este período.

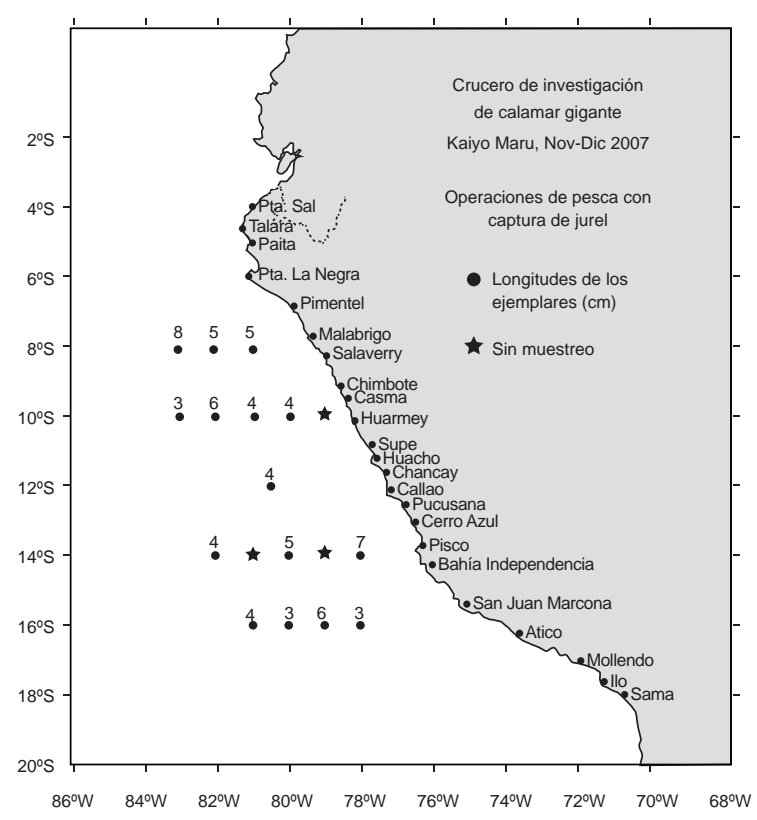

Figura 14.- Distribución de juveniles de jurel T. murphyi obtenidos con Red de media agua Nichimo Larva Catcher. Fuente: IMARPE 2008.

Figure 14. - Distribution of juveniles of Jack mackerel T. murphyi obtained with Nichimo Midwater Net Larva Catcher. Fuente:IMARPE 2008.

En la última década se viene presentando un amplio dominio de las ACF retrayendo la ESCC, situación que ha causado cambios en las áreas de pesca, en la estructura latitudinal por tamaños y en las áreas de desove de T. murphyi. Las áreas de pesca, a partir del ańo 2000 se desplazaron de la zona norte hacia los $17^{\circ} \mathrm{S}$ hasta el ańo 2007 , a partir del cual se ubican en la zona central, tal como observamos en la Figura 9. Comportamiento similar observamos con las biomasas acústicas para este mismo periodo frio. En cuanto al desove durante este periodo frio, observamos un desplazamiento del área principal del desove hacia el norte, tal como lo demuestra la captura de juveniles de T. murphyi (3-8 cm LT) mediante una red de media agua Nichimo Larva Catcher durante el Crucero Kaiyo Maru, de noviembre a diciembre 2007 (Fig. 14). De acuerdo con la serie de tiempo disponible del IGS de T. murphyi, este desove fue exitoso, el cual no se ha repetido en años posteriores al 2007, dando lugar a una cohorte de T. murphyi que ha soportado las capturas de los últimos años.

En cuanto a la distribución latitudinal de los tamaños, para el periodo enero-mayo 2011, la Figura 15, nos permite apreciar que en enero, febrero y marzo la pesquería se inició al norte entre los grados 06 y $08^{\circ} \mathrm{S}$ y las capturas tuvieron tallas entre 28 y 30 $\mathrm{cm}$. En tanto que de abril a mayo, la pesquería se realizó entre los $11^{\circ} \mathrm{S}$ y $14^{\circ} \mathrm{S}$ con tallas entre 31 y $32 \mathrm{~cm}$, evidenciando una progresión de tallas de norte a sur lo que indicaría que en este último periodo los reclutamientos se estarían produciendo en este sentido; es decir de norte a sur.

La importancia de la dinámica de las masas de agua frente a la costa peruana y su relación con la disponibilidad de T. murphyi se corrobora una vez más en la distribución de las áreas de pesca y la dominancia de esta especie en las capturas industriales del año 2008, especialmente en marzo. Esas capturas están relacionadas con la aproximación de los frentes subtropical y/o ecuatorial hacia la costa peruana (Fig. 16). 


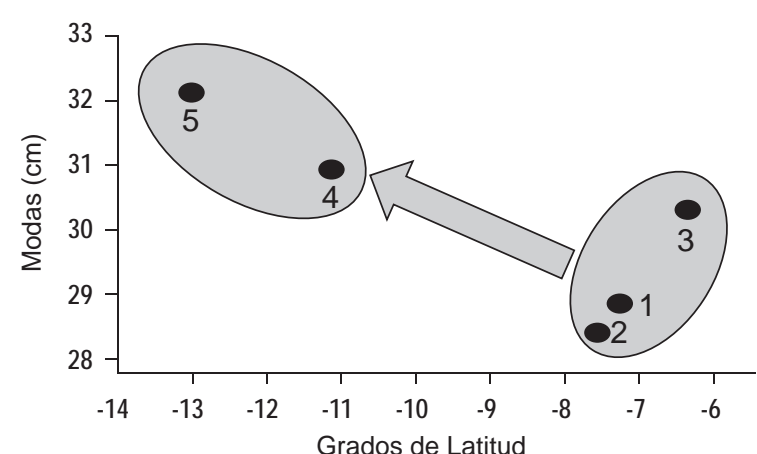

Figura 15.- Modas por grados de latitud de jurel T. murphyi en el mar peruano (enero - mayo 2011, números 1 a 5 ) (Espino, com. pers.).

Figure 15. - Mode by degrees latitude of Jack mackerel T. murphyi in the Peruvian sea (January - May 2011, numners 1 to 5) (Espino, pers. com.).

En síntesis, de este estudio podemos confirmar que el hábitat preferido por T. murphyi es el frente oceanográfico de alta productividad (Dioses et al. 1989), formado por las ASS con las ACF. Este frente determina la abundancia, cercanía o profundización del recurso T. murphyi, y probablemente de otras especies pelágicas de importancia comercial. $\mathrm{Al}$ sur de los $6^{\circ} \mathrm{S}$, las aguas con 35.0 a 35.1 ups tienen mayor relación con las ACF típicas $\mathrm{y}$ las ASS, mientras que al norte de los $6^{\circ} \mathrm{S}$, la mezcla se produce entre las ACF típicas, AES y ASS (Morón 2011). Estas mezclas presentan variaciones espacio-temporales durante el año y sobre ellas ejerce un importante rol la intensidad de los vientos alisios que influyen directamente en el afloramiento costero (Morón 2011), así como en la dinámica del ENSO. Estos y otros aspectos deberán ser profundizados para mejorar nuestro entendimiento del comportamiento de especies como el T. murphyi.

\section{Literatura citada}

Chávez F.P., J. Ryan,S.E. Lluch-Cota \& M. Niquen. 2003. From anchovies to sardines and back: Multidecadal change in the Pacific Ocean. Review: Climate. Science 299: 217-221. DOI: 10.1126/science.1075880

Chávez, F. P., A. Bertrand, R. Guevara-Carrasco, P. Soler, \& J. Csirke. 2008. The northern Humboldt Current System: Brief history, present status and a view towards the future. Progress in Oceanography 79 (2008) 95-105. DOI: 10.1016/j.pocean.2008.10.012

Chirinos J. G. 1992. La pesca del jurel (Trachurus murphyi) y la caballa (Scomber japonicus peruanus) por la Flota Arrastrera Cubana, en el periodo enero 1986-marzo 1987. Tesis para optar el título de ingeniero pesquero. Universidad Nacional Agraria La Molina. Facultad de Pesquería.

Dioses T., V. H. Alarcón, M. E. Nakama \& A. Echevarría. 1989. Desarrollo ovocitario, fecundidad parcial y distribución vertical de los cardúmenes en desove de jurel, Trachurus murphyi (N). En: Memorias del Simposio Internacional de los Recursos Vivos y las Pesquerías en el Pacífico Sudeste. Viña del Mar, 9 - 13 Mayo 1988. Rev. Pacífico Sur (Número Especial), pp. 287 - 294.

Dioses T. 1995. Análisis de la distribución y abundancia de los recursos jurel y caballa frente a la costa peruana. Inst. Mar Perú. Inf. Progresivo 3. pp. 55

Dioses T. \& M. Niquen, 1988. Aspectos generales sobre la biología y pesquería de recurso jurel (Trachurus murphyi) en Perú. En FORUM: Características en el diseńo de una embarcación ideal para la captura de especies pelágicas7. PESCA PERU. $36 \mathrm{pp}$.

Espino M. 2003. Estrategia de gestión oriental para el Pacífico Oriental con especial mención a la pesquería peruana. Tesis para optar el grado de Magíster en Geografía con mención en Ordenamiento y Gestión Ambiental. Unidad de Post Grado, Facultad de Ingeniería Geológica, Minera, Metalúrgica y Geográfica, Universidad Nacional Mayor de San Marcos, Lima. 102 pp.

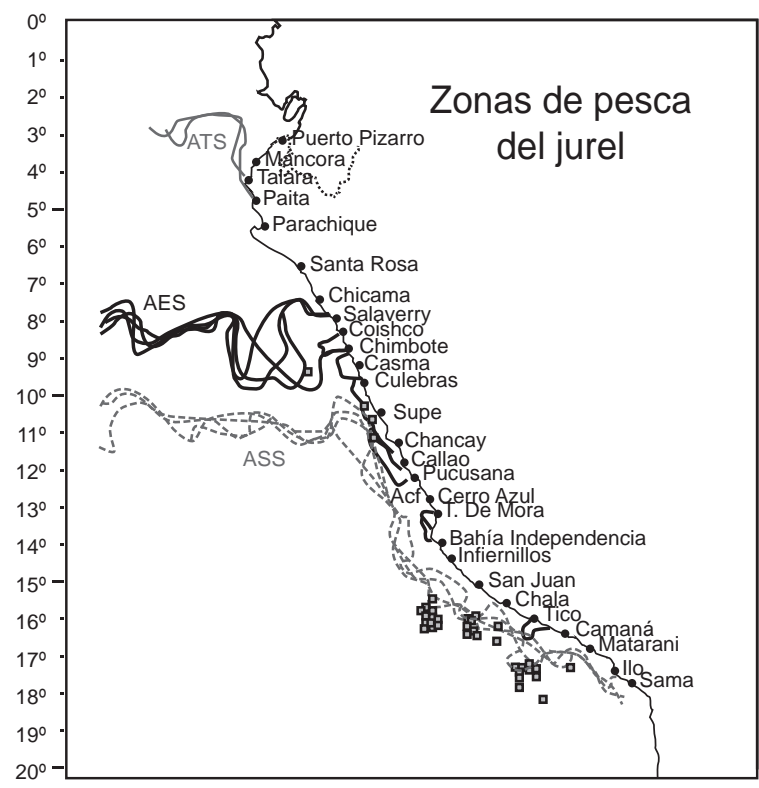

Figura 16. Distribución de las zonas de pesca de jurel T. murphyi y su relación con las masas de agua frente a Perú en marzo 2008. ASS (>35.1 ups) en gris punteado, AES (33.8 a 34.8 ups) en negro entero y ATS ( $>33.8$ ups) en gris entero.

Figure 16. Distribution of Jack mackerel T. murphyi fishing areas and their relationship with the water masses of Peru in March 2008. ASS (> $35.1 \mathrm{psu}$ ) in gray dotted line , AES (from 33.8 to $34.8 \mathrm{psu}$ ) in black line, and ATS (> $33.8 \mathrm{psu}$ ) in gray line.

Espino M. \& C. Yamashiro. 2012. La variabilidad climática y las pesquerías en el Pacífico Suroriental. Lat. Am. J. Aquat. Res., 40(3): 705-721, 2012 Latin American Journal of Aquatic Research 705. International Conference: "Environment and Resources of the South Pacific". P.M. Arana (Guest Editor) DOI: 103856/vol40-issue3-fulltext-18.

Espinoza E., M. Niquen \& Flores R. 2008. Changes in the latitudinal and longitudinal distribution of the aggregations of Jack mackerel (Trachurus murphyi) in the Peruvian sea during $1996-2007$. SPRFMO Chilean Jack Mackerel Workshop, Santiago, Chile, 30 June 4 - July 2008. SPRFMO/CHJMWS Paper 4: 11 pp (http:// www.southpacificrfmo.org/chilean-Jack-mackerel-workshopMeeting-Documents/)

Flores R., J. Tenorio \& N. Domínguez. 2009. Variaciones en la Extensión Sur de la Corriente de Cromwell frente al Perú entre los 3 y $14^{\circ} \mathrm{S}$. Bol. Inst. Mar Perú. 24(1-2):45-58.

Flores R., M. Espino, G. Luque \& J. Quispe. 2013. Patrones de variabilidad ambiental en el mar peruano. En: Csirke J., R. Guevara \& M. Espino (Eds.). Ecología, pesquería y conservación del jurel (Trachurus murphyi) en el Perú. Rev. peru. biol. número especial 20(1): 021 - 028

Ganoza F., A. 1998. Abundancia y comportamiento del jurel (Trachurus picturatus murphyi) a través de proyecciones acústicas de 1983 a 1994. Inf. Inst. Mar Perú 136: 23-47.

Icochea L., A. Chipollini \& M. Niquen. 1989. Análisis de la pesquería de arrastre pelágica en la costa peruana durante 1983-1987 y su relación con el medio ambiente. En: Memorias del Simposio Internacional de Recursos Vivos y las Pesquerías en el Pacífico Sudeste. Viña del Mar, 9 - 13 Mayo 1988. Rev. Pacífico Sur (Número especial), pp. $455-465$.

IMARPE. 2008. Informe ejecutivo del crucero de investigación del calamar gigante BIC Kaiyo Maru 0711-12, noviembre-diciembre 2007. Informe Ejecutivo IMARPE: $14 \mathrm{pp}$.

Morón O. 2011. Climatología de la salinidad superficial del mar frente a la costa peruana. 1960 - 2008. Inf. Inst Mar Perú Vol. 38 N$^{\circ}$ 1: 07 - 39. Enero-marzo 2011.

Santander H. \& R. Flores. 1983. Los desoves y distribución larval de ciertas especies pelágicas y sus relaciones con las variaciones del ambiente marino frente al Perú. FAO Fish Report / FAO Inf. Pesca (291), 3: $835-867$ 